\title{
CHARACTERIZATION OF NONLINEAR SEMIGROUPS ASSOCIATED WITH SEMILINEAR EVOLUTION EQUATIONS
}

\author{
SHINNOSUKE OHARU AND TADAYASU TAKAHASHI
}

\begin{abstract}
Nonlinear continuous perturbations of linear dissipative operators are considered from the point of view of the nonlinear semigroup theory. A general class of nonlinear perturbations of linear contraction semigroups in a Banach space $X$ is introduced by means of a lower semicontinuous convex functional $\rho: X \rightarrow[0, \infty]$ and two notions of semilinear infinitesimal generators of the associated nonlinear semigroups are formulated. Four types of necessary and sufficient conditions are given for a semilinear operator $A+B$ of the class to be the infinitesimal generator of a nonlinear semigroup $\{S(t): t \geq 0\}$ on the domain $C$ of $B$ such that for $x \in C$ the $C$-valued function $S(\cdot) x$ on $[0, \infty)$ provides a unique mild solution of the semilinear evolution equation $u^{\prime}(t)=(A+B) u(t)$ satisfying a growth condition for the function $\mu(u(\cdot))$. It turns out that various types of characterizations of nonlinear semigroups associated with semilinear evolution equations are obtained and, in particular, a semilinear version of the Hille-Yosida theorem is established in a considerably general form.
\end{abstract}

\section{INTRODUCTION}

This paper is concerned with nonlinear semigroups which provide mild solutions of semilinear evolution equations in Banach spaces of the form

$$
u^{\prime}(t)=(A+B) u(t), \quad t>0 .
$$

In (DE) the operator $A$ is assumed to be the infinitesimal generator of a linear $\left(C_{0}\right)$-semigroup $\{T(t): t \geq 0\}$ in a Banach space $X$, and $B$ a nonlinear operator from a convex subset $C$ of $X$ into $X$ which is continuous and quasidissipative in a local sense.

The importance of semilinear equations of the type (DE) has constantly been recognized for many years in various branches of mathematical analysis. However most of the literature dealing with such semilinear equations is devoted to the study of existence and various qualitative properties of the solutions. So

Received by the editors March 24, 1987 and, in revised form, October 6, 1987.

1980 Mathematics Subject Classification (1985 Revision). Primary 58D25, 47H20; Secondary $47 \mathrm{H} 17$.

Key words and phrases. Nonlinear perturbations of linear operators, semilinear evolution equation, mild solution, nonlinear semigroup, full infinitesimal generator, range condition, local quasidissipativity.

The first author's research was supported by the Grant-in-Aid for Scientific Research from the Ministry of Education of Japan. 
far many kinds of sufficient conditions have been given for semilinear equations of the type (DE) to possess global solutions, but it seems to the authors that very little is known about necessary and sufficient conditions on $A$ and $B$ for the solutions of (DE) to exist in a global sense. This problem is important from both theoretical and practical points of view, and it is interesting to discuss a general class of nonlinear perturbations of linear $\left(C_{0}\right)$-semigroups in this direction. In this paper we interpret the above-mentioned problem as a characterization problem of a nonlinear semigroup which provides "mild" solutions of (DE) satisfying an appropriate growth condition, and we discuss the characterization of such nonlinear semigroups in terms of the corresponding "semilinear" infinitesimal generators.

Equation (DE) does not necessarily admit strong solutions and the variation of parameters formula

$$
u(t)=T(t) x+\int_{0}^{t} T(t-s) B u(s) d s, \quad t \geq 0,
$$

is employed to define the generalized solutions that are called mild solutions of (DE). Further, the integral equation (IE) may have only local solutions provided $B$ is locally Lipschitz continuous on $C$ (our assumption on the nonlinear operator $B$ is considerably weaker than the usual local Lipschitz continuity). Here we employ a lower semicontinuous functional $\mu$ on $X$ into $[0, \infty]$ to define the local quasi-dissipativity of $B$ and specify the growth of a solution $u(\cdot)$ of (IE) in terms of the real-valued function $\rho(u(\cdot))$. In case of concrete partial differential equations the use of such a functional $\rho$ corresponds to a priori estimates or energy estimates which ensure the global existence of the solutions as well as their asymptotic properties.

Our objective here is twofold. First, we discuss a new notion of weak solution which is eventually equivalent to the notion of mild solution but more convenient for discussing the characterization problem. These two notions suggest two natural notions of "semilinear" infinitesimal generators of a nonlinear semigroup which provides the mild solutions. It should be mentioned that the ordinary notion of infinitesimal generator is inadequate to characterize such semigroups. In view of these observations we introduce a general class of nonlinear perturbations of linear $\left(C_{0}\right)$-semigroups by using a functional $\mu: X \rightarrow[0, \infty]$ and we establish four types of necessary and sufficient conditions for a semilinear operator $A+B$ of the class to be the semilinear infinitesimal generator of a nonlinear semigroup $\{S(t): t \geq 0\}$ on $C$ such that for $x \in C$ the $C$ valued continuous function $u(t) \equiv S(t) x$ on $[0, \infty)$ is a unique mild solution of (DE) satisfying a certain growth condition on the function $p(u(\cdot))$. The sufficiency of each condition yields a distinct generation theorem for nonlinear semigroups associated with semilinear evolution equations, while the necessity implies that the infinitesimal generator of a nonlinear semigroup $\{S(t)\}$ on $C$ as mentioned above is densely defined in $C$ and exactly equal to the semilinear operator $A+B$. The sufficiency can be proved by applying recent results on 
time-dependent evolution equations in Banach spaces. The proof of the necessity needs a new idea and the central part of this paper is devoted to this argument. It should be noted here that convexity conditions on $C$ and $\mu$ are necessary for the proof. Consequently, four types of characterizations of the nonlinear semigroup associated with (DE) are obtained, and it turns out that a semilinear Hille-Yosida theorem is established in a considerably general form.

\section{Main Results}

In this section we outline the main results of this paper. Let $X$ be a Banach space with norm $|\cdot|$ and $C$ a fixed subset of $X$. Let $\{T(t): t \geq 0\}$ be a linear $\left(C_{0}\right)$-semigroup in $X, A$ the infinitesimal generator with domain $D(A)$, and let $B$ be a nonlinear operator from $C$ into $X$. In this paper we are concerned with the semilinear operator $A+B$ with domain $D(A) \cap C$ which is generically called a nonlinear perturbation of a $\left(C_{0}\right)$-semigroup.

Let $x \in C$ and consider the initial-value problem for (DE):

$$
u^{\prime}(t)=(A+B) u(t), \quad t>0 ; \quad u(0)=x .
$$

An $X$-valued continuous function $u(\cdot)$ on $[0, \infty)$ is called a mild solution of (1.1) if $u(t) \in C$ for $t \geq 0, B u(\cdot)$ is continuous on $[0, \infty)$, and $u(\cdot)$ satisfies

$$
u(t)=T(t) x+\int_{0}^{t} T(t-s) B u(s) d s \text { for } t \geq 0 .
$$

On the other hand, a function $u(\cdot):[0, \infty) \rightarrow C$ is said to be a weak solution of $(1.1)$ if $u(\cdot)$ and $B u(\cdot)$ are continuous on $[0, \infty)$ and for every $f \in D\left(A^{*}\right)$ the function $\langle u(\cdot), f\rangle$ is of class $C^{1}[0, \infty)$ and satisfies the equation

$$
(d / d t)\langle u(t), f\rangle=\left\langle u(t), A^{*} f\right\rangle+\langle B u(t), f\rangle \text { for } t \geq 0 .
$$

We are concerned with a nonlinear semigroup $\{S(t): t \geq 0\}$ on $C$ such that for each $x \in C$ the function $u(t) \equiv S(t) x$ gives a mild solution of (1.1). Equations (1.2) and (1.3) suggest two notions of "semilinear" infinitesimal generators of $\{S(t)\}$. Relation (1.2) implies

$$
\lim _{h \downarrow 0} h^{-1}(S(h) x-T(h) x)=B x \text { for } x \in C,
$$

and formula (1.3) (together with Proposition 2.4) implies

$$
\lim _{h \downarrow 0}\left\langle h^{-1}(S(h) x-x), f\right\rangle=\left\langle x, A^{*} f\right\rangle+\langle B x, f\rangle \text { for } x \in C \text { and } f \in D\left(A^{*}\right) .
$$

A semilinear operator $A+B$ is called the full infinitesimal generator of $\{S(t)\}$ if (1.4) holds; and $A+B$ is said to be the weak tangential operator of $\{S(t)\}$ if (1.5) holds. For the semilinear infinitesimal generators as mentioned above we obtain the following result (Theorem 3.1).

Theorem 1. The following four statements are equivalent.

(i) For $x \in C$ the function $S(\cdot) x$ is a mild solution of (1.1). 
(ii) For $x \in C$ the function $S(\cdot) x$ is a weak solution of (1.1).

(iii) $A+B$ is the full infinitesimal generator of $\{S(t)\}$.

(iv) $A+B$ is the weak tangential operator of $\{S(t)\}$.

In view of Theorem 1 we introduce a general class of nonlinear perturbations of $\left(C_{0}\right)$-semigroups such that to each semilinear operator of the class there corresponds a nonlinear semigroup which provides a unique mild solution of (1.1) for each $x \in C$. We consider a proper lower semicontinuous functional $\mu: X \rightarrow[0, \infty]$ such that $C$ is contained in the effective domain $D(\mu) \equiv\{x \in$ $X: \mu(x)<\infty\}$ and the level set $C_{\alpha} \equiv\{x \in C: \mu(x) \leq \alpha\}$ is closed in $X$ for $\alpha \geq 0$. We say that a semilinear operator $A+B$ is of the class $\mathfrak{S}(C, \rho)$ if $A$ and $B$ satisfy the three conditions below:

(H1) $A-\omega I$ is dissipative in $X$ for some $\omega \in \mathbf{R}$.

(H2) For each $\alpha>0, B$ is continuous on $C_{\alpha}$. $C_{\alpha}$

(H3) For each $\alpha>0$ there is $\omega_{\alpha} \in \mathbf{R}$ such that $B-\omega_{\alpha} I$ is dissipative on

Because of the localized condition (H3) problem (1.1) may have only local mild solutions, and it is necessary to consider the growth of mild solutions. Here we specify the growth of a mild solution $u(\cdot)$ of $(1.1)$ by means of the function $\mu(u(\cdot))$. A nonnegative continuous function $g$ on $[0, \infty)$ is called a comparison function if for each $\alpha \geq 0$ the initial-value problem

$$
w^{\prime}(t)=g(w(t)), \quad t>0 ; \quad w(0)=\alpha
$$

has a maximal solution $m(t ; \alpha)$ on $[0, \infty)$. We choose such a comparison function $g$ and consider global mild solutions $u(\cdot)$ of (1.1) satisfying

$$
\rho(u(t)) \leq m(t ; \mu(x)) \text { for } t \geq 0 .
$$

For mild solutions satisfying the growth condition (1.6) we obtain the following uniqueness theorem (Proposition 5.1).

Theorem 2. Let $A+B$ be of the class $\mathfrak{S}(C, \mu)$ and let $\{S(t): t \geq 0\}$ be a nonlinear semigroup on $C$ such that for $x \in C$ the function $u(\cdot) \equiv S(\cdot) x$ satisfies (1.2) and (1.6). Then for each $\alpha>0$ and each $\tau>0$ we have

$$
|S(t) x-S(t) y| \leq e^{\omega(\alpha, \tau) t}|x-y| \text { for } t \in[0, \tau] \text { and } x, y \in C_{\alpha},
$$

where $\omega(\alpha, \tau)=\omega+\omega_{\beta}$ and $\beta=m(\tau ; \alpha)$. Moreover, for each $x \in C$, the function $S(\cdot) x$ gives a unique mild solution of $(1.1)$ on $[0, \infty)$ satisfying (1.6).

By a continuous semigroup on $C$ is meant a semigroup of (possibly nonlinear) continuous operators from $C$ into itself. A continuous semigroup $\{S(t)\}$ on $C$ is said to be locally equi-Lipschitz continuous on $C$ if for each $\alpha>0$ and each $\tau>0$ there is a constant $\omega(\alpha, \tau)$ for which (1.7) holds. Theorem 2 states that a continuous semigroup $\{S(t)\}$ on $C$ satisfying the growth condition (1.6) is uniquely determined by the operators $A$ and $B$, and that $\{S(t)\}$ is locally equi-Lipschitz continuous on $C$. We then state a part of the main result (Theorem 6.3). 
Theorem 3. Let $A+B$ be a semilinear operator of the class $\mathfrak{S}(C, \mu)$ and suppose that $C$ is convex in $X$ and $\mu$ is convex on $X$. Then the following two conditions are equivalent:

(I) There exists a continuous semigroup $\{S(t): t \geq 0\}$ on $C$ such that for each $t \geq 0$ and each $x \in C$

(I.a) $S(t) x=T(t) x+\int_{0}^{t} T(t-s) B S(s) x d s$, and

(I.b) $\mu(S(t) x) \leq m(t ; \mu(x))$.

(V) $D(A) \cap C$ is dense in $C$; for $\alpha>0$ and $\varepsilon>0$ there exists $\lambda_{0}=$ $\lambda_{0}(\alpha, \varepsilon)>0$ such that to each $x \in C_{\alpha}$ and each $\lambda \in\left(0, \lambda_{0}\right)$ there corresponds an element $x_{\lambda} \in D(A) \cap C$ satisfying

(V.a) $x_{\lambda}-\lambda(A+B) x_{\lambda}=x$, and

(V.b) $\mu\left(x_{\lambda}\right) \leq \rho(x)+\lambda[g(\mu(x))+\varepsilon]$.

Therefore, the infinitesimal generator of $\{S(t)\}$ satisfying (I.a) and (I.b) is exactly the semilinear operator $A+B$ and the domain $D(A) \cap C$ is dense in $C$.

It is not possible to characterize a nonlinear semigroup $\{S(t)\}$ satisfying (I.a) and (I.b) in terms of the infinitesimal generator in the usual sense (see $\S \S 3$ and 7). But combining Theorems 1 and 3, we obtain the following semilinear Hille-Yosida theorem (Theorem 7.2).

Theorem 4. A semilinear operator $A+B$ of the class $\mathfrak{S}(C, \mu)$ is the full infinitesimal generator of a continuous semigroup $\{S(t)\}$ on $C$ satisfying $\mu(S(t) x) \leq$ $m(t ; \rho(x))$ for $t \geq 0$ and $x \in C$ iff the domain $D(A) \cap C$ is dense in $C$ and for $\alpha>0$ and $\varepsilon>0$ there exists $\lambda_{0} \equiv \lambda_{0}(\alpha, \varepsilon)>0$ such that for $\beta \geq \alpha+\lambda\left[\max _{0 \leq \xi \leq \alpha} g(\xi)+\varepsilon\right]$ we have $R\left(I-\lambda\left(A+B_{\beta}\right)\right) \supset C_{\alpha}$ and

$$
\rho\left(\left(I-\lambda\left(A+B_{\beta}\right)\right)^{-1} x\right) \leq \rho(x)+\lambda[g(\rho(x))+\varepsilon]
$$

for $\lambda \in\left(0, \lambda_{0}\right)$ and $x \in C_{\alpha}$, where $B_{\beta}$ denotes the restriction of $B$ to $C_{\beta}$.

The semigroup $\{S(t)\}$ is determined through the exponential formula

$$
S(t) x=\lim _{n \rightarrow \infty}\left(I-\frac{t}{n}\left(A+B_{\beta}\right)\right)^{-n} x, \quad x \in C_{\alpha}, t \in[0, \tau],
$$

where $\alpha>0, \tau>0$ and $\beta>m(\tau ; \alpha)$. If in particular $\rho \equiv 0$ and $g(r) \equiv 0$ in the above theorem, then $B$ is continuous and quasi-dissipative on all of $C$ (i.e., $B-\omega I$ is dissipative for some $\omega \in \mathbf{R})$ and condition $(\mathrm{V})$ reads as follows: $A+B$ is densely defined in $C$ and satisfies the range condition $R(I-\lambda(A+B)) \supset C$ for $\lambda>0$ sufficiently small. Finally, in $\S 7$, a variety of observations on the above-mentioned results are given.

\section{Generalized SOlutions OF SEMILINEAR EVOLUTION EQUATIONS}

In this section we introduce two notions of generalized solutions to (DE) and investigate basic properties of the generalized solutions.

Let $X$ be a real Banach space with norm $|\cdot|$. The dual space of $X$ is denoted by $X^{*}$. For $x \in X$ and $f \in X^{*}$ the value of $f$ at $x$ is written as $\langle x, f\rangle$. The 
duality mapping of $X$ is the mapping $F$ from $X$ into the power set of $X^{*}$ which assigns to each $y \in X$ the weak-star compact convex set $F(y) \equiv\{f \in$ $\left.X^{*}:\langle y, f\rangle=|y|^{2}=|f|^{2}\right\}$. For $x, y \in X$ the symbols $\langle x, y\rangle_{i}$ and $\langle x, y\rangle_{s}$ stand for the infimum and the supremum of $\{\langle x, y\rangle: f \in F(y)\}$, respectively. Given a subset $D$ of $X$, we write $\bar{D}$ for the closure of $D$ and $d(x, D)$ for the distance from $x \in X$ to $D$, i.e., $d(x, D)=\inf \{|x-y|: y \in D\}$. The domain and the range of a (possibly nonlinear) operator $B$ in $X$ are denoted by $D(B)$ and $R(B)$, respectively. The identity operator on $X$ is denoted by $I$.

Let $\{T(t): t \geq 0\}$ be a semigroup of linear operators of class $\left(C_{0}\right)$ on $X$. The infinitesimal generator $A$ of $\{T(t)\}$ is defined through the relation $A x=$ $\lim _{h \perp 0} h^{-1}(T(h) x-x)$ on the set $D(A)$ of all elements $x \in X$ for which the limits on the right-hand side exist. It is well known (see $[3,5,15,17])$ that $A$ is a densely defined closed linear operator in $X$. For the adjoint operator $A^{*}$ of $A$ we have $\langle A x, f\rangle=\left\langle x, A^{*} f\right\rangle$ for $x \in D(A)$ and $f \in D\left(A^{*}\right)$.

We begin with three elementary results which were effectively applied to discuss weak solutions of linear evolution equations with inhomogeneous terms in the work of Ball [1].

Lemma 2.1. If $v, z \in X$ and $\langle z, f\rangle=\left\langle v, A^{*} f\right\rangle$ for all $f \in D\left(A^{*}\right)$, then $v \in D(A)$ and $z=A v$.

Proof. Suppose that the ordered pair $[v, z]$ does not belong to the graph $G(A)$ of $A$. Since $G(A)$ is closed in the product space $X \times X$, the Hahn-Banach theorem would assert that there is a pair $f, g \in X^{*}$ such that $\langle z, f\rangle+\langle v, g\rangle \neq 0$ and $\langle A x, f\rangle+\langle x, g\rangle=0$ for $x \in D(A)$. Hence $\langle A x, f\rangle=\langle x,-g\rangle$ for $x \in D(A)$, which means that $f \in D\left(A^{*}\right)$ and $g=-A^{*} f$. Therefore it would follow that $\langle z, f\rangle \neq\left\langle v, A^{*} f\right\rangle$. This is a contradiction, and we conclude that $[v, z] \in G(A)$. Q.E.D.

Lemma 2.2. An $X$-valued function $v(\cdot)$ on $[0, \infty)$ with $v(0)=0$ is a $C^{1}$ solution of the equation $v^{\prime}(t)=A v(t)$ on $[0, \infty)$ if and only if $v(t) \equiv 0$ on $[0, \infty)$.

Proof. The function $v(t) \equiv 0$ is a trivial $C^{1}$-solution of the initial-value problem. Conversely, let $v(\cdot)$ be a $C^{1}$-solution of the initial-value problem. Then

$$
(\partial / \partial s) T(s) v(t-s)=A T(s) v(t-s)-T(s) A v(t-s)=0
$$

for $0 \leq s \leq t$, and so

$$
v(t)=T(0) v(t)-T(t) v(0)=-\int_{0}^{t}\left(\frac{\partial}{\partial s}\right) T(s) v(t-s) d s=0
$$

for $t \geq 0$. Q.E.D.

Lemma 2.3. For $x \in X$ and $f \in D\left(A^{*}\right)$, the real-valued function $\langle T(\cdot) x, f\rangle$ is of class $C^{1}[0, \infty)$ and $(d / d t)\langle T(t) x, f\rangle=\left\langle T(t) x, A^{*} f\right\rangle$ for $t \geq 0$. 
Proof. Let $t \geq 0$ and let $h \neq 0$ be such that $t+h \geq 0$. Then

$$
\begin{aligned}
& h^{-1}(\langle T(t+h) x, f\rangle-\langle T(t) x, f\rangle) \\
& \quad=h^{-1}\left\langle A \int_{t}^{t+h} T(s) x d s, f\right\rangle=h^{-1} \int_{t}^{t+h}\left\langle T(s) x, A^{*} f\right\rangle d s
\end{aligned}
$$

for $f \in D\left(A^{*}\right)$. Passing to the limit as $h \rightarrow 0$, we see that the derivative of $\langle T(\cdot) x, f\rangle$ coincides with the continuous function $\left\langle T(\cdot) x, A^{*} f\right\rangle$. Q.E.D.

We now introduce a notion of semilinear operator with which we are concerned in this paper. Let $A$ be the infinitesimal generator of a $\left(C_{0}\right)$-semigroup $\{T(t): t \geq 0\}$ on $X, C$ a subset of $X$, and let $B$ be a nonlinear operator from $C$ into $X$. If $D(A) \cap C \neq \varnothing$, then the sum $A+B$ defines an operator in $X$ with domain $D(A+B)=D(A) \cap C$. In this case we call the operator $A+B$ a semilinear operator in $X$. The domain $D(A) \cap C$ is empty in general, but we use the symbol $A+B$ to denote the semilinear operator determined by a pair of operators $A$ and $B$ even though the domain may be empty.

We then consider the initial-value problem for the semilinear evolution equation $(\mathrm{DE})$ :

$$
u^{\prime}(t)=(A+B) u(t), \quad t>0 ; \quad u(0)=x \in C .
$$

Problem (2.1) does not necessarily admit strong solutions in the usual sense even if $x \in D(A), C=X$, and $B$ is continuous on all of $X$. Hence it is required to think of appropriate notions of generalized solutions of (2.1), and the following is known as the most natural notion of such generalized solutions.

Definition 2.1. An $X$-valued continuous function $u(\cdot)$ on $[0, \infty)$ is said to be a mild solution of $(2.1)$ if $u(t) \in C$ for $t \geq 0, B u(\cdot)$ is continuous on $[0, \infty)$, and $u(\cdot)$ satisfies

$$
u(t)=T(t) x+\int_{0}^{t} T(t-s) B u(s) d s, \quad t \geq 0 .
$$

We also employ the following notion of generalized solution which is in fact equivalent to that of mild solution.

Definition 2.2. An $X$-valued continuous function $u(\cdot)$ on $[0, \infty)$ is said to be a weak solution of $(2.1)$ if $u(t) \in C$ for $t \geq 0, B u(\cdot)$ is continuous on $[0, \infty)$, $u(0)=x$, the function $\langle u(\cdot), f\rangle$ is of class $C^{1}[0, \infty)$, for every $f \in D\left(A^{*}\right)$, and

$$
(d / d t)\langle u(t), f\rangle=\left\langle u(t), A^{*} f\right\rangle+\langle B u(t), f\rangle \text { for } t \geq 0 .
$$

The above notion is a modified version of Ball's weak solution [1] and is useful for later arguments. Using Ball's argument, we obtain

Proposition 2.4. Let $x \in C$ and let $u(\cdot)$ be an $X$-valued continuous function on $[0, \infty)$ with $u(0)=x$. Then $u(\cdot)$ is a mild solution of $(1.1)$ if and only if it is a weak solution of (1.1). 
Proof. Suppose that $u(\cdot)$ is a mild solution of problem (2.1). Then

$$
\langle u(t), f\rangle=\langle T(t) x, f\rangle+\int_{0}^{t}\langle T(t-s) B u(s), f\rangle d s \text { for } t \geq 0 \text { and } f \in D\left(A^{*}\right) .
$$

Since $B u(\cdot)$ is continuous on $[0, \infty)$, we infer from Lemma 2.3 that for every $f \in D\left(A^{*}\right)$ the real-valued function $\langle u(\cdot), f\rangle$ is of class $C^{1}[0, \infty)$ and (2.4)

$$
\begin{aligned}
\left(\frac{d}{d t}\right)\langle u(t), f\rangle & =\left(\frac{d}{d t}\right)\langle T(t) x, f\rangle+\left(\frac{d}{d t}\right) \int_{0}^{t}\langle T(t-s) B u(s), f\rangle d s \\
& =\left\langle T(t) x, A^{*} f\right\rangle+\int_{0}^{t}\left\langle T(t-s) B u(s), A^{*} f\right\rangle d s+\langle B u(t), f\rangle \\
& =\left\langle u(t), A^{*} f\right\rangle+\langle B u(t), f\rangle
\end{aligned}
$$

for $t \geq 0$. This shows that $u(\cdot)$ is a weak solution of $(2.1)$. Conversely, let $u(\cdot)$ be a weak solution of (2.1) and define

$$
\bar{u}(t)=T(t) x+\int_{0}^{t} T(t-s) B u(s) d s \quad \text { for } t \geq 0 .
$$

Then, in the same way as in the derivation of (2.4), we see that for every $f \in D\left(A^{*}\right),\langle\bar{u}(\cdot), f\rangle$ is of class $C^{1}[0, \infty)$ and $(d / d t)\langle\bar{u}(t), f\rangle=\left\langle\bar{u}(t), A^{*} f\right\rangle+$ $\langle B u(t), f\rangle$ for $t \geq 0$. Hence

$$
(d / d t)\langle\bar{u}(t)-u(t), f\rangle=\left\langle\bar{u}(t)-u(t), A^{*} f\right\rangle
$$

for $t \geq 0$ and $f \in D\left(A^{*}\right)$. Let $z(t)=\bar{u}(t)-u(t)$ for $t \geq 0$. Then $z(0)=0$ and we have $\langle z(t), f\rangle=\left\langle\int_{0}^{t} z(s) d s, A^{*} f\right\rangle$ for $t \geq 0$ and $f \in D\left(A^{*}\right)$. Therefore $v(t) \equiv \int_{0}^{t} z(s) d s \in D(A)$ and $(d / d t) v(t)=z(t)=A v(t)$ for $t \geq 0$ by Lemma 2.1. Since $v(\cdot)$ is of class $C^{1}([0, \infty) ; X)$, it follows from Lemma 2.2 that $v(t) \equiv 0$ on $[0, \infty)$ and hence $\bar{u}(t)=u(t)$ for $t \geq 0$. This means that $u(\cdot)$ is a mild solution of (2.1). Q.E.D.

The following useful fact may be an oral tradition, although we have no references which contain the explicit mention.

Proposition 2.5. Let $\omega \in R, x \in C$, and let $u(\cdot)$ be an $X$-valued continuous function on $[0, \infty)$ such that $u(t) \in C$ for $t \geq 0$ and $B u(\cdot)$ is continuous on $[0, \infty)$. If $u(\cdot)$ satisfies the integral equation

$$
u(t)=e^{-\omega t} T(t) x+\int_{0}^{t} e^{-\omega(t-s)} T(t-s)[B u(s)+\omega u(s)] d s, \quad t \geq 0,
$$

then it is a mild solution of (2.1).

Proof. Suppose that $u(\cdot)$ satisfies (2.5). Let $f \in D\left(A^{*}\right)$. Then we infer from $(2.5)$ that $\langle u(\cdot), f\rangle$ is of class $C^{1}[0, \infty)$ and $(d / d t)\langle u(t), f\rangle$ is computed as

$$
\begin{aligned}
\left(\frac{d}{d t}\right) & \left\langle e^{-\omega t} T(t) x, f\right\rangle+\left(\frac{d}{d t}\right) \int_{0}^{t}\left\langle e^{-\omega(t-s)} T(t-s)[B u(s)+\omega u(s)], f\right\rangle d s \\
= & \left\langle u(t), A^{*} f-\omega f\right\rangle+\langle B u(t)+\omega u(t), f\rangle=\left\langle u(t), A^{*} f\right\rangle+\langle B u(t), f\rangle
\end{aligned}
$$


for $t \geq 0$. By Proposition 2.4, this implies that $u(\cdot)$ is a mild solution of (2.1). Q.E.D.

Remark 2.1. It follows from Proposition 2.5 that $u(\cdot)$ is a mild solution of $(2.1)$ if and only if for some $\omega \in R$ it is a mild solution of the initial-value problem

$$
u^{\prime}(t)=[(A-\omega I)+(B+\omega I)] u(t), \quad t>0 ; \quad u(0)=x \in C .
$$

This fact is also convenient for the discussion on the relatively continuous perturbations of analytic semigroups. It is possible to extend Proposition 2.5 to the case in which the semigroup $\left\{e^{-\omega t} T(t)\right\}$ is replaced by the semigroups generated by bounded linear perturbations of $A$.

Remark 2.2. As will be seen later, the variation of parameters formula (2.2) is convenient for verifying the unicity of solutions, while the weak form (2.3) of equation $(\mathrm{DE})$ is useful for investigating the generators of nonlinear semigroups associated with semilinear evolution equations of the type (DE).

\section{INFINITESIMAL GENERATORS OF NONLINEARLY PERTURBED $\left(C_{0}\right)$-SEMIGROUPS}

In this section we consider nonlinear semigroups which provide mild solutions of semilinear evolution equations of the type (DE) and discuss the infinitesimal generators of such nonlinear semigroups.

Let $A$ be the infinitesimal generator of a $\left(C_{0}\right)$-semigroup $\{T(t): t \geq 0\}$ on $X$ and $B$ a nonlinear operator from a subset $C$ of $X$ into $X$. Suppose for the moment that for each $x \in C$ the initial-value problem (2.1) has a unique mild solution $u(\cdot ; x)$ on $[0, \infty)$. Then one can define operators $S(t), t \geq 0$, in $X$ by

$$
S(t) x=u(t ; x) \text { for } t \geq 0 \text { and } x \in C .
$$

The operators $S(t)$ map $C$ into itself and are necessarily nonlinear. Also, they have the two properties below:

(S1) $S(0) x=x, S(t+s) x=S(t) S(s) x$ for $s, t \geq 0$ and $x \in C$.

(S2) For each $x \in C$, the $X$-valued function $S(\cdot) x$ is continuous over $[0, \infty)$.

The first property is obtained through the uniqueness of mild solutions and is called the semigroup property; the second property states that $S(t)$ depends continuously upon the parameter $t$.

By a nonlinear semigroup on $C$ is meant a one-parameter family $\{S(t): t \geq$ $0\}$ of nonlinear operators from $C$ into itself with the above-mentioned properties (S1) and (S2). If in particular a semigroup on $C$ provides mild solutions of (DE) in the sense of (3.1), we call it the nonlinear semigroup on $C$ associated with the semilinear evolution equation (DE).

Let $\{S(t)\}$ be a nonlinear semigroup on $C$ associated with (DE). Then

$$
S(t) x=T(t) x+\int_{0}^{t} T(t-s) B S(s) x d s \quad \text { for } t \geq 0 \text { and } x \in C .
$$


From this we see that $\lim _{h \downarrow 0} h^{-1}(S(h) x-x)$ exists if and only if $x \in D(A) \cap C$, and that

$$
\lim _{h \downarrow 0} h^{-1}(S(h) x-x)=(A+B) x \quad \text { for } x \in D(A) \cap C .
$$

These propositions make sense provided that $D(A) \cap C \neq \varnothing$. This means that $A+B$ gives exactly the infinitesimal generator of $\{S(t)\}$ in the usual sense. Hence it might be natural to expect that condition (3.2) for a semigroup $\{S(t)\}$ is equivalent to the condition that $D(A) \cap C$ is dense in $C$ and (3.3) holds for the semilinear operator $A+B$. In fact, as shown in Proposition 6.2, condition (3.2) implies the denseness in $C$ of the domain $D(A) \cap C$ of the infinitesimal generator $A+B$ under the assumption that $C$ is convex and $A+B$ is of a certain class of semilinear operators. However, as we mention in the next paragraph, it seems that the converse does not hold in general.

First we observe that a semilinear operator $A+B$ is not uniquely represented and may be decomposed into a different form $A^{\prime}+B^{\prime}$ in such a way that $A^{\prime}$ is no longer the infinitesimal generator of a $\left(C_{0}\right)$-semigroup on $X, D\left(B^{\prime}\right) \varsubsetneqq C$, and $B^{\prime}$ loses the continuity even if $B$ itself is continuous over $C$. In this sense the combination of (3.3) and the denseness of $D(A) \cap C$ in $C$ yields only a single nonlinear operator and does not necessarily provide the exact form of semilinear operator $A+B$. Accordingly, it seems to be insufficient to think of only the infinitesimal generator in the usual sense in order to discuss the characterization of a nonlinear semigroup associated with (DE).

Accordingly, it is crucial to specify the infinitesimal generator as a semilinear operator $A+B$. Here we introduce two notions of "semilinear" infinitesimal generators. First relation (3.2) implies

$$
\lim _{h \downarrow 0} h^{-1}(S(h) x-T(h) x)=B x \text { for } x \in C .
$$

Further, formula (3.3) together with Proposition 2.4 implies

$$
\lim _{h \downarrow 0}\left\langle h^{-1}(S(h) x-x), f\right\rangle=\left\langle x, A^{*} f\right\rangle+\langle B x, f\rangle \quad \text { for } x \in C \text { and } f \in D\left(A^{*}\right) .
$$

It should be noted that relations (3.4) and (3.5) hold on all of $C$ and make sense even if $D(A+B)=D(A) \cap C=\varnothing$. Relation (3.5) may be interpreted as follows: The vector field generated by $A+B$ is tangential in a weak sense to the continuous curve $S(\cdot) x$ in $X$ for each $x \in C$. These facts lead us to the following definitions.

Definition 3.1. Let $\{S(t): t \geq 0\}$ be a nonlinear semigroup on $C$ such that $B S(\cdot) x$ is continuous on $[0, \infty)$ for each $x \in C$. Then $A+B$ is said to be the full infinitesimal generator of $\{S(t)\}$ if $(3.4)$ holds.

Definition 3.2. Let $\{S(t)\}$ be as in Definition 3.1. $A+B$ is said to be the weak tangential operator to $\{S(t)\}$ if (3.5) holds. 
A nonlinear semigroup $\{S(t)\}$ on $C$ associated with (DE) can be characterized in terms of full infinitesimal generator, weak tangential operator and weak solution in the following way.

Theorem 3.1. Let $\{S(t): t \geq 0\}$ be a nonlinear semigroup on $C$ such that $B S(\cdot) x$ is continuous on $[0, \infty)$ for each $x \in C$. The following are equivalent:

(i) $S(t) x=T(t) x+\int_{0}^{t} T(t-s) B S(s) x d s$ for $t \geq 0$ and $x \in C$.

(ii) $\lim _{h \downarrow 0} h^{-1}(S(h) x-T(h) x)=B x$ for $x \in C$.

(iii) $\lim _{h \downarrow 0}\left\langle h^{-1}(S(h) x-x), f\right\rangle=\left\langle x, A^{*} f\right\rangle+\langle B x, f\rangle$ for $x \in C$ and $f \in$ $D\left(A^{*}\right)$.

(iv) $(d / d t)\langle S(t) x, f\rangle=\left\langle S(t) x, A^{*} f\right\rangle+\langle B S(t) x, f\rangle$ for $t \geq 0, x \in C$, and $f \in D\left(A^{*}\right)$.

(v) $\int_{0}^{t} S(s) x d s \in D(A)$ and

$$
S(t) x=x+A \int_{0}^{t} S(s) x d s+\int_{0}^{t} B S(s) x d s \text { for } t>0 \text { and } x \in C \text {. }
$$

Proof. It is obvious that (i) implies (ii). Suppose that (ii) holds. Let $x \in C$ and $f \in D\left(A^{*}\right)$. Then we have

$$
\begin{aligned}
\left\langle h^{-1}(S(h) x-x), f\right\rangle & =\left\langle h^{-1}(S(h) x-T(h) x), f\right\rangle+\left\langle h^{-1}(T(h) x-x), f\right\rangle \\
& =\left\langle h^{-1}(S(h) x-T(h) x), f\right\rangle+\left\langle h^{-1} \cdot \int_{0}^{h} T(s) x d s, A^{*} f\right\rangle .
\end{aligned}
$$

Therefore we obtain (iii) by taking the limits as $h \downarrow 0$ of both sides of the above identity and applying (ii). Assume that (iii) is valid. Using the semigroup property, we get

$$
\begin{aligned}
\left(d^{+} / d t\right)\langle S(t) x, f\rangle=\left\langle S(t) x, A^{*} f\right\rangle+\langle B S(t) x, f\rangle \\
\\
\quad \text { for } t \geq 0, x \in C, \text { and } f \in D\left(A^{*}\right)
\end{aligned}
$$

where the left side denotes the right-hand derivative of $\langle S(\cdot) x, f\rangle$. But the right side of the above relation is continuous in $t \geq 0$, and so the function $\langle S(\cdot) x, f\rangle$ turns out to be of class $C^{1}[0, \infty)$ and the left side can be replaced by the ordinary derivative of $\langle S(\cdot) x, f\rangle$. This means that (iv) holds. It is easy to see that (iv) is equivalent to (v). Finally, by Proposition 2.4, (iv) implies (i). Q.E.D.

Remark 3.1. Condition (v) above states that $u(t ; x) \equiv S(t) x$ satisfies (DE) for a.e. $t \geq 0$ and gives a strong solution of problem $(2.1)$ provided that $A u(\cdot ; x)$ is Bochner integrable over bounded subintervals of $[0, \infty)$. There is an example of semigroups $\{S(t)\}$ associated with semilinear equations of type (DE) such that $S(t) x \notin D(A)$ for $t>0$ and $x \in C$, although it should be noted that $\int_{0}^{t} S(s) x d s \in D(A)$ for all $t>0$ and all $x \in C$.

\section{Nonlinear perturbations of LiNear $\left(C_{0}\right)$-Semigroups}

In this section we introduce a general class (denoted hereinafter $\mathfrak{S}(C, \rho)$ ) of semilinear operators with which we are mainly concerned in this paper. We 
show that to each semilinear operator of the class there corresponds a unique nonlinear semigroup which provides mild solutions to the associated semilinear evolution equation. In order to introduce the class of operators we employ a proper, lower semicontinuous functional $\mu$ on $X$ into $[0, \infty]$. In concrete problems for nonlinear partial differential equations such functionals often arise and play an important role in deriving a priori estimates or various energy estimates for the solutions, and they are also useful for specifying the growth order and investigating asymptotic properties of the solutions. For instance the $L^{\infty}$-norm $\mu(\cdot)=|\cdot|_{\infty}$ of summable functions on a domain $\Omega$ in $\mathbf{R}^{n}$ defines such a functional on the usual Lebesgue space $L^{1}(\Omega)$; the functional which assigns to each element $v$ of the Lebesgue space $L^{\infty}(\Omega)$ the smallest Lipschitz constant $\mu(v)=\operatorname{Lip}(v)$ gives a typical example of such functionals on $L^{\infty}(\Omega)$; and the total variation $\mu(\cdot)=\operatorname{Var}(\cdot)$ of summable functions on $\Omega$ is another useful functional on $L^{1}(\Omega)$.

In a system $(X, \mu)$ of a Banach space $X$ and a functional $\mu$ as mentioned above we introduce the following class of semilinear operators. Let $C$ be a subset of $X$. Without loss of generality we may assume that $C$ is contained in the effective domain $D(\mu)=\{x \in X: \mu(x)<\infty\}$ of $\mu$ (if it is not the case, we take $D(\mu) \cap C$ instead of $C)$. By a semilinear operator of the class $\mathfrak{S}(C, \rho)$ we mean the sum $A+B$ of a linear operator $A$ in $X$ and a nonlinear operator $B$ from $C$ into $X$ satisfying the following conditions:

(H1) $A$ is the infinitesimal generator of a $\left(C_{0}\right)$-semigroup $\{T(t): t \geq 0\}$ on $X$ such that $|T(t) x| \leq e^{\omega t}|x|$ for $t \geq 0, x \in X$, and some $\omega \in R$.

(H2) For each $\alpha>0$ the level set $C_{\alpha}=\{x \in C: \rho(x) \leq \alpha\}$ in $C$ is closed and $B$ is continuous on $C_{\alpha}$.

(H3) For each $\alpha>0$ there exists $\omega_{\alpha} \in R$ such that $B-\omega_{\alpha} I$ is dissipative on $C_{\alpha}$ in the sense that

$$
\langle B x-B y, x-y\rangle_{i} \leq \omega_{\alpha}|x-y|^{2} \text { for } x, y \in C_{\alpha} .
$$

In particular, we write $\mathfrak{S}(C)$ for $\mathfrak{S}(C, \mu)$ provided $\rho(x) \equiv 0$. In case $\rho \equiv 0$, condition (H2) means that $C$ itself is closed and $B$ is continuous on all of $C$.

A few remarks on the class $\mathfrak{S}(C, \rho)$ are in order. Let $A+B$ be of the class $\mathfrak{S}(C, \rho)$. Then by $(\mathrm{H} 1) A-\omega I$ is strictly dissipative in the sense that

$$
\langle A x, x\rangle_{s} \leq \omega|x|^{2} \text { for } x \in D(A) \text {. }
$$

Hence if $D(A) \cap C_{\alpha} \neq \varnothing$, then $A+B-\left(\omega+\omega_{\alpha}\right) I$ is dissipative on $D(A) \cap C_{\alpha}$. As is well known, for each $\lambda>0$ with $\lambda \omega<1$ the resolvent $(I-\lambda A)^{-1}$ exists as a bounded linear operator on $X$ and satisfies

$$
\left|(I-\lambda A)^{-1} x\right| \leq(1-\lambda \omega)^{-1}|x| \text { for } x \in X .
$$

On the other hand, condition (H3) implies that

$$
|x-y| \leq\left(1-\lambda \omega_{\alpha}\right)^{-1}|(x-\lambda B x)-(y-\lambda B y)|
$$

for $\alpha>0, x, y \in C_{\alpha}$, and $\lambda>0$ with $\lambda \omega_{\alpha}<1$. 
We now consider the initial-value problem (2.1) for a semilinear operator $A+B$ of the class $\mathfrak{S}(C, \mu)$. Because of the localized condition (H3) on $B$, (2.1) may admit only local mild solutions. Hence it is necessary to consider the growth order of mild solutions in order to discuss global mild solutions of (2.1), and here we think of specifying the growth of a mild solution $u(\cdot)$ by means of the real-valued function $\rho(u(\cdot))$.

Let $g$ be a nonnegative continuous function on $[0, \infty)$ such that for each $\alpha \geq 0$ the initial-value problem

$$
w^{\prime}(t)=g(w(t)), \quad t>0 ; \quad w(0)=\alpha
$$

has a maximal solution $m(t ; \alpha)$ on $[0, \infty)$. In what follows, we fix such a function $g$ and investigate global mild solutions $u(\cdot)$ of (2.1) satisfying

$$
\rho(u(t)) \leq m(t ; \mu(x)), \quad t \geq 0 .
$$

The function $g$ is sometimes called a comparison function or else a uniqueness function. In view of the localized condition (H3) and the growth condition of the form (4.2), we can deal with a broad class of semilinear evolution equations. In practice, the function $g$ must be chosen in accordance with properties of the function space $X$ as well as the operators $A$ and $B$ under consideration. A typical example of comparison functions is the linear function $g(r)=a r$, $r>0, a$ being a positive constant, and in this case $m(t ; \alpha)=\alpha e^{a t}$ for $\alpha \geq 0$ and $t \geq 0$. For a detailed discussion of this particular case, see the authors' previous paper [11].

We then show that mild solutions $u(\cdot)$ of (2.1) satisfying (4.2) are uniquely determined by and depend continuously upon initial data in each $C_{\alpha}$.

Uniqueness Theorem. Let $A+B$ be a semilinear operator of the class $\mathfrak{S}(C, \rho)$. Then given $x \in C$ there exists at most one mild solution $u(\cdot)$ of (2.1) satisfying (4.2).

Proof. Let $\alpha>0, \tau>0, \beta=m(\tau ; \alpha)$, and $\omega_{\beta}$ denote the constant given for the $\beta$ by condition (H3). Let $x, y \in C_{\alpha}$ and let $u(\cdot), v(\cdot)$ be the corresponding global mild solutions of (2.1) satisfying (4.2). Then $u(t), v(t) \in C_{\beta}$ for $t \in[0, \tau]$. Since $B-\omega_{\beta} I$ is dissipative on $C_{\beta}$, we have

$$
\left(1-h \omega_{\beta}\right)|u(t+h)-v(t+h)| \leq|(I-h B) u(t+h)-(I-h B) u(t+h)|
$$

for $t \in[0, \tau)$ and $h>0$ with $t+h \leq \tau$. But $(I-h B) u(t+h)$ can be written as

$$
T(h) u(t)+\int_{t}^{t+h}[T(t+h-s) B u(s)-B u(t+h)] d s
$$


and $(I-h B) v(t+h)$ is also written in the same way. Hence we have

$$
\begin{aligned}
\left(1-h \omega_{\beta}\right)|u(t+h)-v(t+h)| \\
\leq e^{\omega h}|u(t)-v(t)|+\int_{t}^{t+h}|T(t+h-s) B u(s)-B u(t+h)| d s \\
\quad+\int_{t}^{t+h}|T(t+h-s) B v(s)-B v(t+h)| d s
\end{aligned}
$$

for $t \in[0, \tau)$ and $h \in(0, \tau-t]$. Since

$$
\lim _{h \downarrow 0} \frac{1}{h} \int_{t}^{t+h}|T(t+h-s) B u(s)-B u(t+h)| d s=0
$$

and (4.4) holds for $v(\cdot)$ as well, (4.3) implies

$$
D^{+}|u(t)-v(t)| \leq\left(\omega+\omega_{\beta}\right)|u(t)-v(t)| \text { for } t \in[0, \tau),
$$

where $D^{+} \varphi(t)$ stands for the Dini upper right derivative of an $\mathbf{R}$-valued function $\varphi$ on $[0, \tau)$ at $t$. Solving the differential inequality (4.5), one obtains

$$
|u(t)-v(t)| \leq e^{\left(\omega+\omega_{\beta}\right) t}|x-y| \text { for } t \in[0, \tau] \text { and } x, y \in C_{\alpha} .
$$

From this we obtain the desired assertion. Q.E.D.

Estimate (4.6) not only proves the uniqueness of mild solutions, but also it shows the Lipschitz continuous dependence (in a local sense) of mild solutions on initial-data. We have employed the differential inequality (4.5) to get (4.6); it is possible to derive (4.6) for mild solutions by different methods (see [14, $\S 5.3]$ ). By Proposition 2.4, (4.6) also holds for every pair of weak solutions of (2.1) with (4.2). But it would be an interesting problem to give a direct proof of the uniqueness of weak solutions.

\section{GeNERATION OF NONLINEAR SEMIGROUPS ASSOCIATED WITH (DE)}

In this section we discuss two sufficient conditions for a semilinear operator $A+B$ of the class $\mathfrak{S}(C, \mu)$ to generate a nonlinear semigroup $\{S(t): t \geq 0\}$ on $C$ satisfying

$$
\begin{gathered}
S(t) x=T(t) x+\int_{0}^{t} T(t-s) B S(s) x d s \text { for } t \geq 0 \text { and } x \in C, \\
\mu(S(t) x) \leq m(t ; \mu(x)) \text { for } t \geq 0 \text { and } x \in C .
\end{gathered}
$$

Notice that the integral on the right side of (5.1) is taken in the sense of Bochner and makes sense for every $x \in C$ since $B S(\cdot) x \in C([0, \infty) ; X)$ for $x \in C$ by condition (H2) and (5.2).

A nonlinear semigroup $\{S(t): t \geq 0\}$ on $C$ satisfying (5.1) and (5.2) is Lipschitz continuous on $\mu$-bounded sets in the following sense. 
Proposition 5.1. Let $A+B$ be of the class $\mathfrak{S}(C, \rho)$ and let $\{S(t): t \geq 0\}$ be a nonlinear semigroup on $C$ satisfying (5.1) and (5.2). Then for each $\alpha>0$ and $\tau>0$ there is a number $\omega(\alpha, \tau)$ such that

$$
|S(t) x-S(t) y| \leq e^{\omega(\alpha, \tau) t}|x-y| \text { for } t \in[0, \tau] \text { and } x, y \in C_{\alpha} .
$$

Moreover, for each $x \in C$, the function $S(\cdot) x$ on $[0, \infty)$ gives a unique mild solution of (2.1) satisfying (4.2).

The above proposition is a restatement of the Uniqueness Theorem given in the preceding section. From (4.6) we infer that $\omega(\alpha, \tau)$ appearing in (5.3) may be taken as $\omega+\omega_{\beta}$, where $\beta=m(\tau ; \alpha)$.

Let $A+B$ be a semilinear operator of the class $\mathfrak{S}(C, \rho)$ and consider the following two "subtangential" conditions.

(R1) For each $x \in C$ there exist a null sequence $\left\{h_{n}\right\}$ of positive numbers and a sequence $\left\{x_{n}\right\}$ in $C$ such that

(1.a) $\lim _{n \rightarrow \infty} h_{n}^{-1}\left|T\left(h_{n}\right) x+h_{n} B x-x_{n}\right|=0$, and

(1.b) $\lim \sup _{n \rightarrow \infty} h_{n}^{-1}\left[\rho\left(x_{n}\right)-\rho(x)\right] \leq g(\rho(x))$.

(R2) For each $x \in C$ there exist a null sequence $\left\{h_{n}\right\}$ of positive numbers and a sequence $\left\{x_{n}\right\}$ in $D(A) \cap C$ such that

(2.a) $\lim _{n \rightarrow \infty} h_{n}^{-1}\left|x_{n}-h_{n}(A+B) x_{n}-x\right|=0$,

(2.b) $\lim \sup _{n \rightarrow \infty} h_{n}^{-1}\left[\rho\left(x_{n}\right)-\rho(x)\right] \leq g(\rho(x))$, and

(2.c) $\lim _{n \rightarrow \infty}\left|x_{n}-x\right|=0$.

Condition (1.a) may be called an "explicit" subtangential condition, while condition (2.a) may be called an "implicit" subtangential condition.

Remark 5.1. Condition (2.c) implies that $D(A) \cap C$ is dense in $C$. Conversely, if $D(A) \cap C$ is dense in $C$, then condition (2.c) follows from (2.a) and (2.b). In fact, let $x \in C$ and let $\left\{h_{n}\right\},\left\{x_{n}\right\}$ be sequences satisfying (2.a) and (2.b). Let $\varepsilon>0, y \in D(A) \cap C$, and assume that $|y-x| \leq \varepsilon$. Choose $\alpha>0$ so that $x, y \in C_{\alpha}$ and the sequence $\left\{x_{n}\right\}$ satisfying (2.a) and (2.b) belong to $C_{\alpha}$. Since $A+B-\left(\omega+\omega_{\alpha}\right) I$ is dissipative on $D(A) \cap C_{\alpha}$, we have

$$
\begin{aligned}
\left|x_{n}-x\right| \leq & \left|x_{n}-y\right|+|y-x| \\
\leq & \left(1-h_{n}\left(\omega+\omega_{\alpha}\right)\right)^{-1}\left|\left(I-h_{n}(A+B)\right) x_{n}-\left(I-h_{n}(A+B)\right) y\right|+|y-x| \\
\leq & \left(1-h_{n}\left(\omega+\omega_{\alpha}\right)\right)^{-1}\left[\left|x_{n}-h_{n}(A+B) x_{n}-x\right|+|y-x|+h_{n}|(A+B) y|\right] \\
& +|y-x|
\end{aligned}
$$

for $n$ sufficiently large, where $\omega$ and $\omega_{\alpha}$ denote the numbers given by (H1) and (H3), respectively. Hence $\limsup _{n \rightarrow \infty}\left|x_{n}-x\right| \leq 2|y-x| \leq 2 \varepsilon$. Since $\varepsilon>0$ is arbitrary, this shows that (2.c) holds.

Remark 5.2. Since $(I-\lambda A)^{-1} x \rightarrow x$ as $\lambda \downarrow 0$ for $x \in X$ and $B$ is continuous on each $C_{\alpha}$, it is seen that condition (R2) is equivalent to the following condition: 
(R3) For each $x \in C$ there exist a null sequence $\left\{h_{n}\right\}$ of positive numbers and a sequence $\left\{x_{n}\right\}$ in $D(A) \cap C$ such that

(3.a) $\lim _{n \rightarrow \infty} h_{n}^{-1}\left|x_{n}-h_{n} A x_{n}-\left(x+h_{n} B x\right)\right|=0$, and

(3.b) $\lim \sup _{n \rightarrow \infty} h_{n}^{-1}\left[\rho\left(x_{n}\right)-\rho(x)\right] \leq g(\rho(x))$.

In comparison with conditions (1.a) and (2.a), condition (3.a) may be called a "semi-implicit" subtangential condition.

By applying the recent results on time-dependent evolution equations of the form

$$
u^{\prime}(t)=(A+B(t)) u(t), \quad t>0,
$$

we obtain the following generation theorem.

Theorem 5.2. Suppose that either of (R1) and (R2) is satisfied. Then there exists a nonlinear semigroup $\{S(t): t \geq 0\}$ on $C$ satisfying (5.1) and (5.2).

Proof. In view of the uniqueness theorem it suffices to show that for any positive number $\tau>0$ and any element $z \in C$ there exists an $X$-valued continuous function $u(\cdot)$ on $[0, \tau]$ satisfying the properties below:

$$
\begin{gathered}
u(t) \in C, \quad t \in[0, \tau], \\
u(t)=T(t) z+\int_{0}^{t} T(t-s) B u(s) d s, \quad t \in[0, \tau], \\
\mu(u(t)) \leq m(t ; \rho(z)), \quad t \in[0, \tau] .
\end{gathered}
$$

Let $\tau>0$ and $z \in C$. We first recall (see [8]) that there is a number $\zeta \equiv$ $\zeta(\tau, \mu(z))>0$ such that for $\varepsilon \in(0, \zeta]$ the initial-value problem

$$
w^{\prime}(t)=g(w(t))+\varepsilon, \quad t>0 ; \quad w(0)=\rho(z)
$$

has a maximal solution $m_{\varepsilon}(t ; \mu(z))$ on $[0, \tau]$. Let $\varepsilon \in(0, \zeta]$. Set $\alpha=$ $m_{\varepsilon}(\tau ; \rho(z))$ and let $\omega_{\alpha}$ denote the constant given by (H3). Also, for each $t \in[0, \tau]$, we write $\mathscr{D}(t)$ for the set $\left\{x \in C: \mu(x) \leq m_{\varepsilon}(t ; \mu(z))\right\}$ and define an operator $B(t)$ from $\mathscr{D}(t)$ into $X$ by $B(t) x=B x$ for $x \in \mathscr{D}(t)$. Then the following are valid for the operators $B(t)$ :

(i) Each of $\mathscr{D}(t)$ is closed and $\mathscr{D}(s) \subset \mathscr{D}(t)$ for $0 \leq s \leq t \leq \tau$.

(ii) $B(t) x$ is continuous with respect to $(t, x)$ in $\mathscr{D} \equiv \bigcup_{0 \leq s \leq \tau}\{s\} \times \mathscr{D}(s)$.

(iii) For each $t \in[0, \tau], B(t)-\omega_{\alpha} I$ is dissipative on $\mathscr{D}(t)$.

We first suppose that (R1) holds. Let $x \in C$, let $\left\{h_{n}\right\}$ be a null sequence of positive numbers, and let $\left\{x_{n}\right\}$ be a sequence in $C$. Then

$$
\lim _{n \rightarrow \infty} h_{n}^{-1}\left(x_{n}-T\left(h_{n}\right) x\right)=B x
$$

if and only if

$$
\lim _{n \rightarrow \infty} h_{n}^{-1}\left(x_{n}-\exp \left(-\omega h_{n}\right) T\left(h_{n}\right) x\right)=(B+\omega I) x
$$

for every $\omega \in T$. Hence, in view of Proposition 2.5, we may assume without loss of generality that condition (H1) holds with $\omega=0$. Let $t \in[0, \tau)$ and 
$x \in \mathscr{D}(t)$. Then by (R1) there exist sequences $\left\{h_{n}\right\}$ and $\left\{x_{n}\right\}$ satisfying (1.a) and (1.b). Hence, by the continuity of $g$ and condition (1.b) we have

$$
\begin{aligned}
\mu\left(x_{n}\right) \leq & \rho(x)+h_{n}[g(\mu(x))+\varepsilon / 2] \\
\leq & \rho(x)+\int_{0}^{h_{n}} g\left(m_{\varepsilon}(s ; \rho(x))\right) d s \\
& +\int_{0}^{h_{n}}\left[g(\mu(x))-g\left(m_{\varepsilon}(s ; \mu(x))\right)+\varepsilon / 2\right] d s \\
\leq & m_{\varepsilon}\left(h_{n} ; \rho(x)\right)
\end{aligned}
$$

for $n$ sufficiently large. Since $\mu(x) \leq m_{\varepsilon}(t ; \mu(z))$, it follows that

$$
\rho\left(x_{n}\right) \leq m_{\varepsilon}\left(h_{n} ; m_{\varepsilon}(t ; \rho(z))\right) \leq m_{\varepsilon}\left(t+h_{n} ; \rho(z)\right)
$$

for $n$ sufficiently large. From this and (1.a) we infer that

(iv) $\liminf _{h \downarrow 0} h^{-1} d(T(h) x+h B(t) x, \mathscr{D}(t+h))=0$ for $t \in[0, \tau)$ and $x \in$ $\mathscr{D}(t)$.

By virtue of facts (i)-(iv) mentioned above, we can apply the results of Iwamiya [4] and Pavel [14] to conclude that there exists a function $u(\cdot) \in$ $C([0, \tau] ; X)$ such that $u(t) \in \mathscr{D}(t)$ and (5.6) holds for $t \in[0, \tau]$. Since $u(t) \in C_{\alpha}$ for $t \in[0, \tau]$, it follows from the unicity of $u(\cdot)$ that $u(\cdot)$ is independent of $\varepsilon \in(0, \zeta]$. The fact that $u(t) \in \mathscr{D}(t)$ for $t \in[0, \tau]$ means that

$$
\mu(u(t)) \leq m_{\varepsilon}(t ; \mu(z)) \text { for } t \in[0, \tau] \text { and } \varepsilon \in(0, \zeta] .
$$

Further, $\lim _{\varepsilon \downarrow 0} m_{\varepsilon}(t ; \mu(z))=m(t ; \mu(z))$ for $t \in[0, \tau]$, and so the function $u(\cdot)$ satisfies (5.7). This completes the proof under condition $(\mathrm{R} 1)$.

Next, suppose that (R2) holds. By the same reasoning as in the above argument we may assume again that $(\mathrm{H} 1)$ holds with $\omega=0$. In this case the operators $A+B(t)$ with domains $D(A+B(t))=D(A) \cap \mathscr{D}(t)$ satisfy

(v) For $s, t \in[0, \tau], x \in D(A+B(s))$, and $y \in D(A+B(t))$,

$$
\langle(A+B(s)) x-(A+B(t)) y, x-y\rangle_{i} \leq \omega_{\alpha}|x-y|^{2} .
$$

Further, in a way similar to the derivation of (iv), we infer from (2.a) and (2.b) that

(vi) $\liminf _{h \downarrow 0} h^{-1} d(x, R(I-h(A+B(t+h))))=0$ for $t \in[0, \tau)$ and $x \in$ $\mathscr{D}(t)$.

In view of facts (i), (v), and (vi), the results of Kobayasi et al . [7] and Pavel [12] can be applied to conclude that the following holds:

(W) There exist a sequence $\left\{\Delta_{n}\right\}$ of partitions of $[0, \tau]$, a sequence $\left\{\varepsilon_{n}(\cdot)\right\}$ of $X$-valued step functions on $(0, \tau]$, and a sequence $\left\{u_{n}(\cdot)\right\}$ of $X$-valued step 
functions on $[0, \tau]$ such that

$\left(\mathrm{w}_{1}\right)$ for every $n \geq 1$,

$$
\begin{gathered}
\Delta_{n}=\left\{0=t_{0}^{n}<t_{1}^{n}<\cdots<t_{N(n)}^{n} \leq \tau\right\}, \\
\varepsilon_{n}(t)=\varepsilon_{k}^{n} \text { for } t \in\left(t_{k-1}^{n}, t_{k}^{n}\right] \text { and } 1 \leq k \leq N(n), \\
u_{n}(0)=x_{0}^{n}=z, \quad u_{n}(t)=x_{k}^{n} \quad \text { for } t \in\left(t_{k-1}^{n}, t_{k}^{n}\right] \text { and } 1 \leq k \leq N(n), \\
\left(t_{k}^{n}-t_{k-1}^{n}\right)^{-1}\left(x_{k}^{n}-x_{k-1}^{n}\right)-\varepsilon_{k}^{n}=\left(A+B\left(t_{k}^{n}\right)\right) x_{k}^{n} \text { for } 1 \leq k \leq N(n) ;
\end{gathered}
$$

$\left(\mathrm{w}_{2}\right) \lim _{n \rightarrow \infty} \max _{1 \leq k \leq N(n)}\left(t_{k}^{n}-t_{k-1}^{n}\right)=0, \lim _{n \rightarrow \infty} t_{N(n)}^{n}=\tau$, and $\lim _{n \rightarrow \infty} \int_{0}^{\tau}\left|\varepsilon_{n}(t)\right| d t=0$;

$\left(w_{3}\right) u_{n}(t)$ converges to some $X$-valued continuous function $u(t)$ on $[0, \tau]$ as $n \rightarrow \infty$ and the convergence is uniform for $t \in[0, \tau]$.

Let $u(\cdot)$ be the function obtained by $\left(\mathrm{w}_{3}\right)$. Then $u(t) \in \overline{D(A) \cap \mathscr{D}(t)} \subset \mathscr{D}(t)$ for $t \in[0, \tau]$. By the same reasoning as in the proof under condition (R1), this implies that $u(\cdot)$ satisfies (5.7). It now remains to show that $u(\cdot)$ satisfies (5.6). To this end, let $f \in D\left(A^{*}\right)$. From ( $\left.\mathrm{w}_{1}\right)$ it follows that

$$
\left\langle x_{k}^{n}, f\right\rangle=\left\langle x_{k-1}^{n}, f\right\rangle+\left(t_{k}^{n}-t_{k-1}^{n}\right)\left[\left\langle x_{k}^{n}, A^{*} f\right\rangle+\left\langle B x_{k}^{n}, f\right\rangle+\left\langle\varepsilon_{k}^{n}, f\right\rangle\right]
$$

for $n \geq 1$ and $1 \leq k \leq N(n)$. Hence an induction argument implies that

$$
\left\langle u_{n}(t), f\right\rangle=\langle z, f\rangle+\int_{0}^{t_{k}^{n}}\left[\left\langle u_{n}(s), A^{*} f\right\rangle+\left\langle B u_{n}(s), f\right\rangle+\left\langle\varepsilon_{n}(s), f\right\rangle\right] d s
$$

for $t \in\left(t_{k-1}^{n}, t_{k}^{n}\right], n \geq 1$, and $1 \leq k \leq N(n)$. Taking any $t \in[0, \tau]$ and passing to the limit as $t_{k}^{n} \rightarrow t$ and $n \rightarrow \infty$, we obtain

$$
\langle u(t), f\rangle=\langle z, f\rangle+\int_{0}^{t}\left[\left\langle u(s), A^{*} f\right\rangle+\langle B u(s), f\rangle\right] d s \quad \text { for } t \in[0, \tau] .
$$

This together with Proposition 2.4 implies that $u(\cdot)$ satisfies (5.6). Thus the proof under condition (R2) is obtained. Q.E.D.

\section{Characterization OF NONLINEARLY PERTURBed $\left(C_{0}\right)$-SEMIGROUPS}

In this section necessary and sufficient conditions are given for a semilinear operator $A+B$ of the class $\mathfrak{S}(C, \rho)$ to generate a nonlinear semigroup $\{S(t): t \geq 0\}$ on $C$ satisfying (5.1) and (5.2). For each $h>0$ and $\tau>0$, we define an operator $J_{h, \tau}$ from $C$ into $X$ by

$$
J_{h, \tau} x=\left(a_{h, \tau}\right)^{-1} \int_{0}^{\tau} e^{-t / h} S(t) x d t \quad \text { for } x \in C,
$$

where $a_{h, \tau}=\int_{0}^{\tau} e^{-t / h} d t=h\left(1-e^{-\tau / h}\right)$. The right-hand side of (6.1) may be regarded as a local Laplace transform of $S(\cdot) x$ and the operators $J_{h, \tau}$ are used to investigate the ranges of the operators $I-\lambda(A+B), \lambda>0$. 
Proposition 6.1. Suppose that both $C$ and $\rho$ are convex, and let $J_{h, \tau}$ be defined by (6.1). Then the operators $J_{h, \tau}$ have the five properties below:

(i) For $h>0, \tau>0$, and $x \in C, J_{h, \tau} x \in D(A) \cap C$ and $(I-h A) J_{h, \tau} x$ can be written as

$$
x+h\left(a_{h, \tau}\right)^{-1} \int_{0}^{\tau} e^{-t / h} B S(t) x d t-h e^{-\tau / h}\left(a_{h, \tau}\right)^{-1}(S(\tau) x-x) .
$$

(ii) $\lim _{h\lrcorner 0} h^{-1}\left|(I-h A) J_{h, \tau} x-(x+h B x)\right|=0$ for $\tau>0$ and $x \in C$.

(iii) $\lim _{h \downarrow 0}\left|J_{h, \tau} x-x\right|=0$ for $\tau>0$ and $x \in C$.

(iv) $\lim \sup _{h \downarrow 0} h^{-1}\left[\rho\left(J_{h, \tau} x\right)-\rho(x)\right] \leq g(\mu(x))$ for $\tau>0$ and $x \in C$.

(v) $\lim _{h \downarrow 0} \mu\left(J_{h, \tau} x\right)=\mu(x)$ for $\tau>0$ and $x \in C$.

Proof. Let $h>0, \tau>0$ and let $J_{h, \tau}$ be defined by (6.1). Let $x \in C$. Then it follows from (5.2) and the convexity of $\rho$ that $J_{h, \tau} x \in C_{\alpha}$ for some $\alpha>0$. To show (i), we employ Theorem 3.1(v) which states that for every $t>0$, $\int_{0}^{t} S(s) x d s \in D(A)$ and $A \int_{0}^{t} S(s) x d s=S(t) x-x-\int_{0}^{t} B S(s) x d s$. Multiplying both sides of this relation by $\left(a_{h, \tau}\right)^{-1} e^{-t / h}$ and integrating the resultant equality with respect to $t$ over $(0, \tau]$, we have

$$
\begin{aligned}
&\left(a_{h, \tau}\right)^{-1} \int_{0}^{\tau}\left[e^{-t / h} A \int_{0}^{t} S(s) x d s\right] d t \\
& \quad=\left(a_{h, \tau}\right)^{-1} \int_{0}^{\tau} e^{-t / h}(S(t) x-x) d t-\left(a_{h, \tau}\right)^{-1} \int_{0}^{\tau}\left[e^{-t / h} \int_{0}^{t} B S(s) x d s\right] d t \\
& \quad=J_{h, \tau} x-x+h e^{-\tau / h}\left(a_{h, \tau}\right)^{-1} \int_{0}^{\tau} B S(s) x d s-h\left(a_{h, \tau}\right)^{-1} \int_{0}^{\tau} e^{-t / h} B S(t) x d t .
\end{aligned}
$$

We apply Theorem 3.1(v) again to replace the third term on the right side by

$$
h e^{-\tau / h}\left(a_{h, \tau}\right)^{-1}\left[S(\tau) x-x-A \int_{0}^{\tau} S(s) x d s\right] \text {. }
$$

On the other hand, the left side can be written as

$A\left[\left(a_{h, \tau}\right)^{-1} \int_{0}^{\tau} e^{-t / h} \int_{0}^{t} S(s) x d s d t\right]=h A J_{h, \tau} x-h e^{-\tau / h}\left(a_{h, \tau}\right)^{-1} A \int_{0}^{\tau} S(t) x d t$,

so that we obtain the first assertion (i). Since $e^{-\tau / h}\left(a_{h, \tau}\right)^{-1} \rightarrow 0$ and

$$
\left(a_{h, \tau}\right)^{-1} \int_{0}^{\tau} e^{-t / h} B S(t) x d t \rightarrow B x \quad \text { as } h \rightarrow 0+,
$$

assertion (ii) is obtained from the relation given in (i). Since

$$
\left|J_{h, \tau} x-x\right| \leq\left|J_{h, \tau} x-(I-h A)^{-1}(x+h B x)\right|+\left|(I-h A)^{-1}(x+h B x)-x\right|
$$

and $\left|(I-h A)^{-1}\right| \leq(1-\omega h)^{-1}$, assertion (iii) is a direct consequence of (ii). We next show that (iv) holds. Let $\tau>0$ and $x \in C$. Since $\mu$ is convex and lower semicontinuous, we see from (5.2) and the definition of $J_{h, \tau} x$ that

$$
\rho\left(J_{h, \tau} x\right) \leq\left(a_{h, \tau}\right)^{-1} \int_{0}^{\tau} e^{-t / h} m(t ; \rho(x)) d t
$$


and

$$
h^{-1}\left[\mu\left(J_{h, \tau} x\right)-\mu(x)\right] \leq h^{-1}\left(a_{h, \tau}\right)^{-1} \int_{0}^{\tau} e^{-t / h}[m(t ; \mu(x))-\mu(x)] d t .
$$

By integration by parts, the right-hand side can be written as

$$
-e^{-\tau / h}\left(a_{h, \tau}\right)^{-1}(m(\tau ; \mu(x))-\mu(x))+\left(a_{h, \tau}\right)^{-1} \int_{0}^{\tau} e^{-t / h} g(m(t ; \mu(x))) d t,
$$

and so we have

$$
\limsup _{h \downarrow 0} h^{-1}\left[\rho\left(J_{h, \tau} x\right)-\rho(x)\right] \leq g(\rho(x))
$$

which proves (iv). Finally, (iii) and (iv) together yield

$$
\rho(x) \leq \liminf _{h \downarrow 0} \rho\left(J_{h, \tau} x\right) \leq \limsup _{h \downarrow 0} \rho\left(J_{h, \tau} x\right) \leq \mu(x),
$$

from which assertion ( $v$ ) follows. Q.E.D.

The next result is an immediate consequence of Proposition 6.1.

Proposition 6.2. Let $A+B$ be of the class $\mathfrak{S}(C, \rho)$ and let $\{S(t): t \geq 0\}$ be a nonlinear semigroup on $C$ satisfying (5.1) and (5.2). If both $C$ and $\mu$ are convex, then

$$
\bigcup_{\alpha>0} \overline{D(A) \cap C_{\alpha}}=C .
$$

In particular, $D(A) \cap C$ is dense in $C$.

We are now in a position to prove the main result of this paper.

Theorem 6.3. Let $A+B$ be a semilinear operator of the class $\mathfrak{S}(C, \mu)$ and suppose that $C$ is convex in $X$ and $\mu$ is convex on $X$. Then the following are equivalent.

(I) There exists a nonlinear semigroup $\{S(t): t \geq 0\}$ on $C$ satisfying

(I.a) $S(t) x=T(t) x+\int_{0}^{t} T(t-s) B S(s) x d s$,

(I.b) $\rho(S(t) x) \leq m(t ; \mu(x))$ for $t \geq 0$ and $x \in C$.

(II) For each $x \in C$ and each $\varepsilon>0$ there exists a pair $\left(h, x_{h}\right) \in(0, \varepsilon] \times C$ such that

(II.a) $\left|T(h) x+h B x-x_{h}\right| \leq h \varepsilon$,

(II.b) $\mu\left(x_{h}\right) \leq \mu(x)+h[g(\rho(x))+\varepsilon]$.

(III) For each $x \in C$ there exist a null sequence $\left\{h_{n}\right\}$ of positive numbers and a sequence $\left\{x_{n}\right\}$ in $C$ such that

(III.a) $\lim _{n \rightarrow \infty} h_{n}^{-1}\left|T\left(h_{n}\right) x+h_{n} B x-x_{n}\right|=0$,

(III.b) $\lim \sup _{n \rightarrow \infty} h_{n}^{-1}\left[\rho\left(x_{n}\right)-\rho(x)\right] \leq g(\mu(x))$.

(IV) For each $x \in C$ there exist a null sequence $\left\{h_{n}\right\}$ of positive numbers and a sequence $\left\{x_{n}\right\}$ in $D(A) \cap C$ such that

(IV.a) $\lim _{n \rightarrow \infty} h_{n}^{-1}\left|\left(x_{n}-h_{n} A x_{n}\right)-\left(x+h_{n} B x\right)\right|=0$,

(IV.b) $\quad \lim \sup _{n \rightarrow \infty} h_{n}^{-1}\left[\rho\left(x_{n}\right)-\mu(x)\right] \leq g(\mu(x))$. 
(V) $D(A) \cap C$ is dense in $C$; given $\alpha>0$ and $\varepsilon>0$ there exists a number $\lambda_{0}=\lambda_{0}(\alpha, \varepsilon)>0$ and to each $x \in C_{\alpha}$ and each $\lambda \in\left(0, \lambda_{0}\right)$ there corresponds an element $x_{\lambda} \in D(A) \cap C$ satisfying

(V.a) $x_{\lambda}-\lambda(A+B) x_{\lambda}=x$,

(V.b) $\mu\left(x_{\lambda}\right) \leq \mu(x)+\lambda[g(\mu(x))+\varepsilon]$.

Proof. It is easy to see that (I) implies (II). In fact, let (I) hold and fix any $x \in C$. Set $x_{h}=S(h) x$ for $h>0$. Then

$$
\begin{aligned}
& \lim _{h \downarrow 0} h^{-1}\left|T(h) x+h B x-x_{h}\right|=\lim _{h \downarrow 0}\left|h^{-1}(S(h) x-T(h) x)-B x\right|=0, \\
& \underset{h \downarrow 0}{\limsup } h^{-1}\left[\rho\left(x_{h}\right)-\rho(x)\right] \leq \limsup _{h \downarrow 0} h^{-1}[m(h ; \rho(x))-\rho(x)]=g(\rho(x)) \text {. }
\end{aligned}
$$

This shows that (II) holds. The implication (II) $\rightarrow$ (III) is obvious. Next, it is seen from Remarks 5.1 and 5.2 that (V) implies (IV). The implication (III) $\rightarrow$ (I) is a restatement of Theorem 5.2. Also, the implication (IV) $\rightarrow$ (I) is obtained from Remark 5.2 and Theorem 5.2. It should be noted that the convexity assumption on $C$ and $\rho$ is not necessary for the proofs of the above implications. We now show that (I) implies (V). Suppose (I) holds. Since $D(A) \cap C$ is dense in $C$ by Proposition 6.2, it suffices to show that the latter half of condition (V) hold. Fix any $\alpha>0$ and $\varepsilon>0$. Then one can choose a number $\delta \in(0,1]$ so that

$$
|g(\xi)-g(\eta)| \leq \varepsilon / 2 \text { for } \xi, \eta \in[0, \alpha+1] \text { with }|\xi-\eta| \leq \delta .
$$

Using the numbers $\alpha, \varepsilon$ and $\delta$, we define

$$
\lambda_{0}=\min \left\{1 / \bar{\omega}, \delta /\left(\max _{0 \leq \xi \leq \alpha+1} g(\xi)+\varepsilon\right)\right\},
$$

where $\bar{\omega}=\max \left\{0, \omega+\omega_{\alpha+1}\right\}$ and $\omega, \omega_{\alpha+1}$ denote the numbers given by conditions (H1) and (H3), respectively. Let $x \in C_{\alpha}, \lambda \in\left(0, \lambda_{0}\right)$, and set

$$
\beta=\mu(x)+\lambda[g(\rho(x))+\varepsilon] .
$$

We wish to show that there exists $x_{\lambda}$ in the set $D(A) \cap C_{\beta}$ satisfying the relation (V.a). Once this is done, then it is concluded that the latter half of $(V)$ is valid. Let $\tau>0, y \in C_{\beta}$, and set

$$
y_{h}=(1-h) J_{\lambda h, \tau} y+h J_{\lambda h, \tau} x \text { for } h \in(0,1],
$$

where the $J_{\lambda h, \tau}$ denote the operators defined by (6.1). Then, by Proposition 6.1 ,

$$
y_{h} \in D(A) \cap C \quad \text { for } h \in(0,1], \quad y_{h} \rightarrow y \quad \text { as } h \rightarrow 0+\text {, }
$$

and

$$
\begin{aligned}
\mid y_{h}- & \lambda h A y_{h}-(y+\lambda h B y-h y+h x) \mid \\
\leq & (1-h)\left|J_{\lambda h, \tau} y-\lambda h A J_{\lambda h, \tau} y-(y+\lambda h B y)\right| \\
& +h\left|J_{\lambda h, \tau} x-\lambda h A J_{\lambda h, \tau} x-(x+\lambda h B x)\right|+\lambda h^{2}|B x-B y| \\
= & \lambda o(h)+\lambda h^{2}|B x-B y| \quad \text { as } h \rightarrow 0+.
\end{aligned}
$$


We then demonstrate that

$$
y_{h} \in C_{\beta} \quad \text { for sufficiently small } h \in(0,1] .
$$

If $p(y)<\beta$, then $(6.8)$ follows from the fact that

$$
\limsup _{h \downarrow 0} \rho\left(y_{h}\right) \leq \limsup _{h \downarrow 0}\left[(1+h) \rho\left(J_{\lambda h, \tau} y\right)+h \mu\left(J_{\lambda h, \tau} x\right)\right]=\rho(y) .
$$

(In the above estimate the last equality is seen from Proposition 6.1(v).) If $p(y)=\beta$, then the application of Proposition 6.1 yields the estimate

$$
\begin{aligned}
\mu\left(y_{h}\right) & \leq(1-h) \mu\left(J_{\lambda h, \tau} y\right)+h \mu\left(J_{\lambda h, \tau} x\right) \\
& \leq(1-h)[\mu(y)+\lambda h(g(\rho(y))+\varepsilon / 4)]+h[\mu(x)+\lambda \varepsilon / 4] \\
& \leq(1-h) \rho(y)+h[\rho(x)+\lambda(g(\mu(y))+\varepsilon / 2)]
\end{aligned}
$$

for $h \in(0,1]$ sufficiently small. From (6.4) and (6.5) we see that $\mu(y)=\beta \leq$ $\alpha+\delta \leq \alpha+1$ and $|\rho(y)-\rho(\dot{x})| \leq \delta$. Hence (6.3) implies $g(\rho(y)) \leq$ $g(\rho(x))+\varepsilon / 2$ and the extreme right-hand side of the above inequality is bounded above by

$$
(1-h) \rho(y)+h[\rho(x)+\lambda(g(\rho(x))+\varepsilon)]=\beta .
$$

This shows that (6.8) is valid. We now denote by $B_{\beta}$ the restriction of the operator $B$ to the set $C_{\beta}$. Then $\lambda B_{\beta}-I+x$ is an operator from $C_{\beta}$ into $X$, where $+x$ denotes the translation by $x$; and we infer from (6.6), (6.7), (6.8) and Remark 5.2 that $\lambda A+\lambda B_{\beta}-I+x$ is a semilinear operator of the class $\mathfrak{S}\left(C_{\beta}\right) \equiv \mathfrak{S}\left(C_{\beta}, 0\right)$ satisfying $\overline{D\left(\lambda A+\lambda B_{\beta}-I+x\right)}=\overline{D(A) \cap C_{\beta}}=C_{\beta}$ and

$$
\lim _{h \downarrow 0} h^{-1} d\left(y, R\left(I-h\left(\lambda A+\lambda B_{\beta}-I+x\right)\right)\right)=0 \text { for } y \in C_{\beta} .
$$

Hence we can apply Theorem 5.2 and Proposition 5.1 (see also [6]) to conclude that there exists a nonlinear semigroup $\left\{S_{\lambda}(t): t \geq 0\right\}$ on $C_{\beta}$ such that

$$
S_{\lambda}(t) y=T(\lambda t) y+\int_{0}^{t} T(\lambda(t-s))\left[\lambda B S_{\lambda}(s) y-S_{\lambda}(s) y+x\right] d s,
$$

$$
\left|S_{\lambda}(t) y-S_{\lambda}(t) z\right| \leq e^{(\lambda \bar{\omega}-1) t}|y-z| \text { for } t \geq 0, y \in C_{\beta} \text { and } z \in C_{\beta},
$$

where $\bar{\omega}=\max \left\{0, \omega+\omega_{\alpha+1}\right\}$. Since $\lambda \bar{\omega}<1,(6.10)$ ensures that $\left\{S_{\lambda}(t)\right\}$ has a common fixed point. Namely, there exists a unique element $x_{\lambda}$ in $C_{\beta}$ such that

$$
S_{\lambda}(t) x_{\lambda}=x_{\lambda} \text { for all } t \geq 0 .
$$

Since $\lambda A+\lambda B_{\beta}-I+x$ is the infinitesimal generator of $\left\{S_{\lambda}(t): t \geq 0\right\}$, it follows from (6.11) that $x_{\lambda} \in D(A) \cap C_{\beta}$ and

$$
\lambda(A+B) x_{\lambda}-x_{\lambda}+x=0 .
$$


This shows that $x_{\lambda}$ satisfies (V.a) and (V.b). Since $x \in C_{\alpha}$ and $\lambda \in\left(0, \lambda_{0}\right)$ are arbitrary, it is concluded that $(\mathrm{V})$ holds under $(\mathrm{I})$. Thus conditions $(\mathrm{I})-(\mathrm{V})$ are equivalent. Q.E.D.

\section{NOTES AND REMARKS}

In this section we give a variety of observations of the results obtained in the previous sections.

7.1. We have shown in Proposition 6.2 that the infinitesimal generator $A+B$ of the semigroup $\{S(t)\}$ on $C$ satisfying (I.a) and (I.b) is densely defined in $C$. However the proof of Theorem 6.3 (more precisely, the argument for the derivation of (6.8)) implies a stronger denseness condition for the domain of $A+B$.

Proposition 7.1. Let $A+B$ be a semilinear operator of the class $\mathfrak{S}(C, \mu)$ and assume that both $C$ and $\rho$ are convex. Let $C_{\alpha} \neq \varnothing$ for some $\alpha \geq 0$ and suppose that condition (I) holds. Then

$$
\overline{D(A) \cap C_{\beta}}=C_{\beta} \text { for } \beta>\alpha \text {. }
$$

7.2. Let $A+B$ be a semilinear operator of the class $\mathfrak{S}(C, \mu)$. If we denote by $B_{\beta}$ the restriction of $B$ to $C_{\beta}$ for $\beta>0$, then condition (V) can be rewritten in the following form:

(R) $D(A) \cap C$ is dense in $C$ and for every $\alpha>0$ and $\varepsilon>0$ there is a number $\lambda_{0}=\lambda_{0}(\alpha, \varepsilon)>0$ such that for $\beta \geq \alpha+\lambda\left[\max _{0 \leq \xi \leq \alpha} g(\xi)+\varepsilon\right]$ we have

$$
\begin{gathered}
R\left(I-\lambda\left(A+B_{\beta}\right)\right) \supset C_{\alpha} \text { for } \lambda \in\left(0, \lambda_{0}\right), \\
\mu\left(\left(I-\lambda\left(A+B_{\beta}\right)\right)^{-1} x\right) \leq \rho(x)+\lambda[g(\rho(x))+\varepsilon] \text { for } \lambda \in\left(0, \lambda_{0}\right) \text { and } x \in C_{\alpha} .
\end{gathered}
$$

Furthermore, it is seen from the proof of Theorem 5.2 that if the "range condition (R)" holds for the semilinear operator $A+B$, then $A+B$ determines a unique nonlinear semigroup $\{S(t)\}$ on $C$ satisfying condition (I) in such a way that for $\alpha>0, \tau>0$ and $\beta>m(\tau ; \alpha)$ the exponential formula

$$
S(t) x=\lim _{n \rightarrow \infty}\left(I-\frac{t}{n}\left(A+B_{\beta}\right)\right)^{-n} x
$$

holds for $t \in[0, \tau]$ and $x \in C_{\alpha}$. Notice that $m_{\varepsilon}(\tau ; \alpha) \leq \beta$ for sufficiently small $\varepsilon>0$. Thus, combining Theorem 3.1 and Theorem 6.3, we obtain the following semilinear Hille-Yosida theorem.

Theorem 7.2. Suppose that $C$ and $\rho$ are convex. Then a semilinear operator $A+B$ of the class $\mathfrak{S}(C, \mu)$ is the full infinitesimal generator of a continuous semigroup $\{S(t)\}$ on $C$ satisfying $\mu(S(t) x) \leq m(t ; \mu(x))$ for $t \geq 0$ and $x \in C$ iff the range condition (R) holds. Moreover, in this case, $\{S(t)\}$ is determined through the exponential formula (7.2).

From the above theorem two results below are deduced. The first result shows that the correspondence between nonlinear semigroups satisfying the growth condition (I.b) and their full infinitesimal generators is one-to-one. 
Corollary 7.3. Let $C$ and $\rho$ be convex. Let $A_{1}+B_{1}$ and $A_{2}+B_{2}$ be two semilinear operators of the the class $\mathfrak{S}(C, \mu)$ and assume that they are the full infinitesimal generators of nonlinear semigroups $\left\{S_{1}(t): t \geq 0\right\}$ and $\left\{S_{2}(t): t \geq\right.$ $0\}$ on $C$ satisfying the growth condition (I.b), respectively. Then $S_{1}(t)=S_{2}(t)$ for $t \geq 0$ iff $A_{1}+B_{1}=A_{2}+B_{2}$.

The second result is a direct consequence of Theorem 7.2, and it may be understood as a semilinear version of the Lumer-Phillips theorem.

Corollary 7.4. Let $A$ be the infinitesimal generator of a $\left(C_{0}\right)$-semigroup $\{T(t): t$ $\geq 0\}$ of linear contractions on $X$. Let $C$ be a closed convex subset of $X$ and let $B$ be a continuous operator from $C$ into $X$ such that $B-\omega I$ is dissipative on $C$ for some $\omega \in \mathbf{R}$. Then $A+B$ is the full infinitesimal generator of a nonlinear semigroup $\{S(t): t \geq 0\}$ on $C$ iff $D(A) \cap C$ is dense in $C$ and $A+B$ satisfies the range condition $R(I-\lambda(A+B)) \supset C$ for $\lambda>0$ with $\lambda \omega<1$. Moreover, in this case, $\{S(t)\}$ is determined via the exponential formula

$$
S(t) x=\lim _{n \rightarrow \infty}(I-(t / n)(A+B))^{-n} x \text { for } t \geq 0 \text { and } x \in C
$$

and it satisfies $\left|S(t) x-x_{0}\right| \leq e^{\omega t}\left(\left|x-x_{0}\right|+t\left|(A+B) x_{0}\right|\right)$ for $t \geq 0, x \in C$ and $x_{0} \in D(A) \cap C$.

7.3. Let $A+B$ be a semilinear operator of the class $\mathfrak{S}(C, \mu)$ and consider the semilinear problem (2.1). Assume that $A$ is dissipative in $X$. In this case one can think of another notion of generalized solution of (2.1) which plays an important role in the theory of nonlinear semigroups in Banach spaces.

Definition 7.1. An $X$-valued continuous function $u(\cdot)$ on $[0, \infty)$ is said to be an integral solution of $(2.1)$ if $u(t) \in C$ for $t \geq 0, B u(\cdot)$ is continuous on $[0, \infty), u(0)=x$ and $u(\cdot)$ satisfies

$$
|u(t)-z|-|u(s)-z| \leq \int_{s}^{t}\langle A z+B u(\xi), u(\xi)-z\rangle_{s} d \xi
$$

for $s, t$ with $0 \leq s \leq t<\infty$ and $z \in D(A)$.

Proposition 7.5. Let $x \in C$ and let $u(\cdot)$ be an $X$-valued continuous function on $[0, \infty)$ with $u(0)=x$. Then $u(\cdot)$ is an integral solution of $(2.1)$ iff it is a mild solution of (2.1).

Proof. Suppose that $u(\cdot)$ is a mild solution of $(2.1)$. Then it is a weak solution of (2.1) by Proposition 2.4. Let $h>0$ and set $u_{h}(t)=h^{-1} \int_{t}^{t+h} u(\xi) d \xi$ and $f_{h}(t)=h^{-1} \int_{t}^{t+h} B u(\xi) d \xi$ for $t \geq 0$. Then we have

$$
\left\langle u(t+h)-u(t)-\int_{t}^{t+h} B u(\xi) d \xi, f\right\rangle=\left\langle\int_{t}^{t+h} u(\xi) d \xi, A^{*} f\right\rangle
$$

for $t \geq 0$ and $f \in D\left(A^{*}\right)$. Hence it follows from Lemma 2.1 that $u_{h}(\cdot)$ satisfies $u_{h}^{\prime}(t)=A u_{h}(t)+f_{h}(t)$ for $t \geq 0$. Since $A$ is strictly dissipative and $\left|u_{h}(\cdot)-z\right|$ is absolutely continuous on compact subintervals of $[0, \infty)$, we get

$$
(d / d t)\left|u_{h}(t)-z\right|=\left\langle u_{h}^{\prime}(t), u_{h}(t)-z\right\rangle_{s} \leq\left\langle A z+f_{h}(t), u_{h}(t)-z\right\rangle_{s}
$$


for $z \in D(A)$ and a.e. $t \geq 0$. Therefore for $s, t$ with $0 \leq s \leq t<\infty$ and $z \in D(A)$ we have

$$
\left|u_{h}(t)-z\right|-\left|u_{h}(s)-z\right| \leq \int_{s}^{t}\left\langle A z+f_{h}(\xi), u_{h}(\xi)-z\right\rangle_{s} d \xi \text { for } h>0 .
$$

Letting $h \downarrow 0$ and using the upper semicontinuity of $\langle\cdot, \cdot\rangle_{s}$, we obtain the integral inequality (7.3). Conversely, let $u(\cdot)$ be an integral solution of (2.1) and set $f(t)=B u(t)$ for $t \geq 0$. Then $u(\cdot)$ is regarded as an integral solution of the initial-value problem

$$
u^{\prime}(t)=A u(t)+f(t), \quad t \geq 0 ; \quad u(0)=x .
$$

Let $\tau>0$ and, for each $n \in \mathbf{N}$, put $h_{n}=\tau / n$ and $t_{i}^{n}=i h_{n}$ for $i=1, \ldots, n$. Since $A$ is $m$-dissipative in $X$, the approximate difference equation

$$
\left(h_{n}\right)^{-1}\left(u_{k}^{n}-u_{k-1}^{n}\right)=A u_{k}^{n}+f\left(t_{k}^{n}\right), \quad k=1, \ldots, n ; \quad u(0)=x,
$$

has a solution $\left(u_{k}^{n}: k=1, \ldots, n\right)$ and is represented as

$$
u_{k}^{n}=\left(I-h_{n} A\right)^{-k} x+h_{n} \sum_{i=1}^{k}\left(I-h_{n} A\right)^{-(k+1-i)} f\left(t_{i}^{n}\right)
$$

for $k=1, \ldots, n$ and $n \in \mathbf{N}$. Hence we have

$$
\lim u_{k}^{n}=u(t ; x) \equiv T(t) x+\int_{0}^{t} T(t-s) f(s) d s \quad \text { for } t \in[0, \tau],
$$

where the limit is taken as $k h_{n} \rightarrow t$ in $[0, \tau]$ and $n \rightarrow \infty$. On the other hand, $\left(u_{k}^{n}\right)$ satisfies the relations

$$
\left(u_{k}^{n}-z\right)-\left(u_{k-1}^{n}-z\right)=h_{n}\left(A u_{k}^{n}-A z\right)+h_{n}\left(A z+f\left(t_{k}^{n}\right)\right),
$$

and so the dissipativity of $A$ implies

$$
\left|u_{k}^{n}-z\right|-\left|u_{k-1}^{n}-z\right| \leq h_{n}\left\langle A z+f\left(t_{k}^{n}\right), u_{k}^{n}-z\right\rangle_{s}
$$

for $k=1, \ldots, n$ and $z \in D(A)$. In view of this and (7.6) we see that the $X$-valued continuous function $u(\cdot ; x)$ is an integral solution of (7.4). But the limit function $u(\cdot ; x)$ of the solutions of $(7.5)$ is unique in the class of integral solutions of $(7.4)$, so that $u(t ; x) \equiv u(t)$ and it is concluded that $u(\cdot)$ becomes the mild solution of $(2.1)$. Q.E.D.

7.4. It is possible to develop a general theory for locally equi-Lipschitz continuous semigroups on convex sets in $X$ which implies the following result related to Corollary 7.3 as its simple consequence.

Proposition 7.6. Let $A+B$ be a semilinear operator of the class $\mathfrak{S}(C, \rho)$. Suppose that $C$ and $\rho$ are both convex, and that $A$ is dissipative in $X$. If $A+B$ is the full infinitesimal generator of a continuous semigroup $\{S(t)\}$ on $C$ satisfying the growth condition

(I.b) $\mu(S(t) x) \leq m(t ; \mu(x))$ for $t \geq 0$ and $x \in C$, 
then $\{S(t)\}$ is the only continuous semigroup on $C$ which satisfies the growth condition (I.b) and whose infinitesimal generator is $A+B$.

For the general result we shall publish it elsewhere.

7.5. Let $A+B$ be a semilinear operator of the class $\mathfrak{S}(C, \mu)$. In order to construct a continuous semigroup on $C$ satisfying condition (I), the explicit subtangential condition (R1) (as stated in $\S 5$ ) or condition (II) (as stated in Theorem 6.3) for $A+B$ can be replaced by the following tangency condition (II) ${ }^{\prime}$ for the nonlinear operator $B$, provided that $A$ is dissipative in $X$, the convex set $C$ is invariant under the linear contraction semigroup $\{T(t)\}$ on $X$ and $\mu(T(t) x) \leq \mu(x)$ for $t \geq 0$ and $x \in C$ :

(II) ' For each $x \in C$ and each $\varepsilon>0$ there exists a pair $\left(h, x_{h}\right) \in(0, \varepsilon] \times C$ such that

(II.a) ${ }^{\prime}\left|x+h B x-x_{h}\right| \leq h \varepsilon$,

(II.b) ${ }^{\prime} \mu\left(x_{h}\right) \leq \rho(x)+h[g(\mu(x))+\varepsilon]$.

In fact, suppose that $T(t)$ maps $C$ into itself for $t \geq 0$, and that $B$ satisfies condition (II) ${ }^{\prime}$. Let $x \in C$ and $\varepsilon>0$. Then there is an $h(\varepsilon) \in(0, \varepsilon]$ such that

$$
|T(h) B x-B x| \leq \varepsilon / 2 \text { for } h \in(0, h(\varepsilon)] .
$$

Further, condition (II)' implies that there is $\left(h, \hat{x}_{h}\right) \in(0, h(\varepsilon)] \times C$ such that $\left|\hat{x}_{h}-(x+h B x)\right| \leq \varepsilon h / 2$ and $\rho\left(\hat{x}_{h}\right) \leq \rho(x)+h[g(\rho(x))+\varepsilon]$. By the assumptions on $\{T(t)\}, x_{h} \equiv T(h) \hat{x}_{h} \in C, \mu\left(x_{h}\right) \leq \mu\left(\hat{x}_{h}\right) \leq \mu(x)+h[g(\mu(x))+\varepsilon]$, and

$$
\begin{aligned}
\left|T(h) x+h B x-x_{h}\right| & \leq\left|T(h)\left(x+h B x-\hat{x}_{h}\right)\right|+h|T(h) B x-B x| \\
& \leq\left|x+h B x-\hat{x}_{h}\right|+h|T(h) B x-B x| \leq h \varepsilon .
\end{aligned}
$$

This shows that condition (II) holds for the semilinear operator $A+B$. Condition (II)' was employed in [9] by Martin.

\section{REFERENCES}

1. J. Ball, Strongly continuous semigroups, weak solutions, and the variation of constants formula, Proc. Amer. Math. Soc. 63 (1977), 370-373.

2. M. Crandall and T. Liggett, Generation of semigroups of nonlinear transformations on general Banach spaces, Amer. J. Math. 93 (1971), 265-298.

3. E. Hille and R. Phillips, Functional analysis and semi-groups, Amer. Math. Soc. Colloq. Publ., vol. 31, Amer. Math. Soc., Providence, R. I., 1957.

4. T. Iwamiya, Global existence of mild solutions to semilinear differential equations in Banach spaces, Hiroshima Math. J. 16 (1986), 499-530.

5. T. Kato, Perturbation theory for linear operators, Springer-Verlag, New York, 1966.

6. Y. Kobayashi, Difference approximation of Cauchy problems for quasi-dissipative operators and generation of nonlinear semigroups, J. Math. Soc. Japan 27 (1975), 640-665.

7. K. Kobayasi, Y. Kobayashi and S. Oharu, Nonlinear evolution operators in Banach spaces, Osaka J. Math. 21 (1984), 281-310.

8. V. Lakshmikantham and S. Leela, Differential and integral inequalities, Academic Press, New York, 1969.

9. R. Martin, Jr., Invariant sets for perturbed semigroups of linear operators, Ann. Mat. Pura Appl. 150 (1975), 221-239. 
10. __ Nonlinear operators and differential equations in Banach spaces, Wiley-Interscience, New York, 1976.

11. S. Oharu and T. Takahashi, Locally Lipschitz continuous perturbations of linear dissipative operators and nonlinear semigroups, Proc. Amer. Math. Soc. 97 (1987), 139-145.

12. N. Pavel, Nonlinear evolution equations governed by $f$-quasi-dissipative operators, Nonlinear Anal. 5 (1981), 449-468.

13. $\ldots$, Semilinear equations with dissipative t-dependent domain perturbations, Israel J. Math. 46 (1983), 103-122.

14. __ Differential equations, flow invariance and applications, Pitman, London, 1984.

15. A. Pazy, Semigroups of linear operators and applications to partial differential equations, Appl. Math. Sci., vol. 44, Springer-Verlag, New York, 1983.

16. G. Webb, Continuous nonlinear perturbations of linear accretive operators in Banach spaces, J. Funct. Anal. 10 (1972), 191-203.

17. K. Yosida, Functional analysis, Springer-Verlag, New York, 1968.

Department of Mathematics, Faculty of Science, Hiroshima University, Hiroshima 730, JAPAN

National Aerospace Laboratory, Chofu, Tokyo 182, Japan 